\title{
Scientific Publishing in Biomedicine: How to Write a Cover Letter?
}

\author{
Zahra Bahadoran (iD) ${ }^{1}$, Parvin Mirmiran (ib ${ }^{1}$, Khosrow Kashfi (id) ${ }^{2}$ and Asghar Ghasemi (id ${ }^{3, *}$ \\ ${ }^{1}$ Nutrition and Endocrine Research Center, Research Institute for Endocrine Sciences, Shahid Beheshti University of Medical Sciences, Tehran, Iran \\ ${ }^{2}$ Department of Molecular, Cellular and Biomedical Sciences, Sophie Davis School of Biomedical Education, School of Medicine, City University of New York, New York, USA \\ ${ }^{3}$ Endocrine Physiology Research Center, Research Institute for Endocrine Sciences, Shahid Beheshti University of Medical Sciences, Tehran, Iran \\ "Corresponding author: Endocrine Physiology Research Center, Research Institute for Endocrine Sciences, Shahid Beheshti University of Medical Sciences, Tehran, Iran. Email: \\ ghasemi@endocrine.ac.ir
}

Received 2021 April 13; Revised 2021 May 29; Accepted 2021 June 12.

\begin{abstract}
A cover (covering) letter is a brief business letter introducing the scientific work alongside the submission process of a manuscript and is required by most scientific peer-review journals. A typical cover letter includes the name of the editor and the journal, date of submission, the characteristics of the manuscript, the importance of the work and its relevance to prospective audiences, declarations such as author agreements, conflicts of interest statement, funding source (s), and ethical statements. The letter also includes the contact information of the corresponding author (s) and may also include suggestions of potential reviewers. Spending enough time to draft an informative, comprehensive, and concise cover letter is quite worthwhile; a poorly drafted one would not persuade the editor that the submitted work is fit for publication and may lead to immediate rejection. Here, we provide a practical guide to draft a well-written, concise, and professional cover letter for a scientific medical paper.
\end{abstract}

Keywords: Cover Letter, Medical Scientific Journals, Scientific Writing, Scientific Publishing

\section{Context}

The Cambridge dictionary defines a cover letter as "a letter that contains information about the thing it is sent with". The cover letter is commonly known as a motivation letter submitted along with the curriculum vitae (CV) or a job application for employment (1) or academic position (2), and it is not clear why and how it was introduced into the scientific field (3). In scientific writing and publishing, a cover/covering letter is a letter to the editor's target journal (4).

Providing a cover letter alongside the submission process is now required by most scientific journals. In fact, some high-quality and prestigious journals pay specific attention to the cover letter (3). Amongst the different steps of the publication process, the cover letter is the last step and is often overlooked (5). One of the most common complaints voiced by editors regarding submitted manuscripts is that the authors neglect to write a wellwritten cover letter, including a statement justifying the importance of their work (6). Missing this opportunity may have unintentional consequences, rejection without further consideration instead of being sent for external peer-review $(5,6)$. Contrary to this view, some believe that the cover letter's content overlaps with the manuscript's abstract and gives mostly redundant information already found within the online submission system (3). The cover letter may also be a "misleading commercial advertisement" where it would not represent the content of the manuscript (3).

Although many editors may not read or seriously consider the cover letters of the submitted manuscripts $(3,4)$, neglecting the importance of the cover letter may be a risk for the authors. Therefore, spending an adequate amount of time to write a coherent and persuasive cover letter is worth it. Following our previous publication on choosing a journal in a new series entitled Scientific Publishing in Biomedicine (7), here, we provided a practical guide to draft a well-written, professional, and concise cover letter needed to be accompanied by an original scientific paper, either with the initial submission or subsequently at revision/resubmitting stage. Since there are subtle differences in writing a cover letter for an original research paper versus a review article or an opinion, some points for drafting a cover letter for such papers are also discussed.

\section{The Function of the Cover Letter}

A cover letter is "a brief business letter", which introduces the submitted manuscript to a prospective editor (s) (8). There are essentially two types of letters; the first 
is the one that is initially submitted with the manuscript (cover letter), and the second is when a revision is being submitted (revised letter). The first letter introduces the work at the initial manuscript submission (9), while the second one is needed following an invitation to revise and resubmit the manuscript. Here, the authors respond to the suggestions/criticisms of the reviewers (10). In this paper, "cover letter" and "second letter" refer to the first/submit letter and the revised letter, respectively.

A well-written cover letter is an effective tool for authors to sell their work to the journal editor and make a "good first impression". A cover letter is a summary that highlights the main points, emphasizes the novelty, and communicates the potential implications of the submitted work (3). A cover letter allows the authors to persuade the editors regarding the novelty/originality and significance of the research in a less formal manner than in the manuscript itself (6). A well-written and informative cover letter helps the journal's editor to be informed about the work and its significance. Regardless of the novelty and significance of the submitted manuscript, editors may miss those points without providing insights in a cover letter (5).

\section{The Content of a Cover Letter}

\subsection{First Cover Letter(Submit Letter)}

One point of view is that the cover letter's content should be covered in the manuscript's abstract (3). A typical cover letter includes the name of editor (s) and the journal, date of submission, the characteristics of the manuscript (i.e., title, type of the manuscript, e.g., review, original, case report), the importance of the work and its relevance to the readership of the journal, verification of the originality of the work, the authors' confirmation that the manuscript is currently submitted only to this journal, declarations and ethical statements, suggested potential reviewers, and contact information of the corresponding author of the submitted work $(5,6)$. Other manuscript characteristics, including the length and number of tables and figures, can also be indicated. If the manuscript belongs to a special issue or is being submitted upon an official invitation from the journal's editorial office, it should also be addressed. The main contents of the first cover letter are described in Table 1.

The most critical element of a cover letter is a "statement of novelty/significance/implication." The authors are advised to carefully write a brief and concise description of their work's impact toward communicating its significance (6). The authors are strongly advised not to copy the abstract into the cover letter and instead explain in their own words the significance of the work and the reason for submitting it to the journal (11). If this information is lacking, the editors may rely on the reviewers who may not appreciate the significance of the work and just focus on the technical issues rather than the scientific value of the work (5). Providing a clear and robust statement of novelty and significance would be more critical for editors and potential reviewers with diverse and interdisciplinary backgrounds (6).

The statements are expected to answer the following questions: (1) why is the work important? (e.g., emphasizing a new measurement, a new diagnostic method or criterion, a newly discovered biological process); and (2) how does the work advance current knowledge in the field? The best approach to answer this question is by describing the current state of knowledge in the field and clarifying how the work provides an added value by answering a previously unanswered question, finding the solution to a problem, or improving existing methods (5). Checking the recently published papers on similar topics in the journal provides new insights for the authors to clarify in the cover letter as to how the manuscript follows the publication trends of the journal and will add something new that would be relevant to the trend (12).

The cover letter is also expected to emphasize why the manuscript will attract the journal's readers (5). The authors also need to consider the journal's Aims and Scope to underscore how the manuscript would fit within the journal's scope and attract potential readers (13). Instead of stating simply that the manuscript is "of interest to the field" or "novel," the authors should address specific aspects of the journal's Aims and Scope statement, e.g., "We believe that this manuscript is appropriate for publication by [journal name] since it... [reference to the journal's aims and scope] (11).

For a review, opinion, or a trends paper, emphasizing the timeline and novelty is needed, as stated by Sacristán, the editor of trends in molecular medicine: "The synthesis and conceptual advance should be particularly stated in terms of what is new and has been trending in the field for the last one to five years". She also recommends that the authors need to provide a future perspective beyond the main take-home message of the manuscript for a trends paper and take a strong and novel stance on a hypothesis or idea for a cover letter of an opinion manuscript (14).

The cover letter must contain some predefined statements, including the "author agreement" statement (13). An "author agreement" is a statement to confirm that "all authors have read and approved the final version of the manuscript being submitted" (8). Furthermore, "the authors warrant that the manuscript is their original work, has not received prior publication and is not under consid- 


\begin{tabular}{|c|c|}
\hline Variables & Values \\
\hline Cover letter header (opening salutation) & Editor (s)' name and position (in bold print); Date of submission (MM-DD-YYYY) \\
\hline \multicolumn{2}{|l|}{ The body of the letter } \\
\hline First paragraph (manuscript's characteristics) & $\begin{array}{l}\text { Title of the manuscript (in bold print); Type of the manuscript, Name of the journal; Address if the } \\
\text { manuscript belongs to a special issue or is being submitted upon an invitation; The length of the } \\
\text { manuscript, the number of tables and figures }\end{array}$ \\
\hline Second paragraph & $\begin{array}{l}\text { The line of research presented in the manuscript; Novelty/significance/implication statement (s) (i.e., } \\
\text { brief explanation regarding the research background of the study, the question answered in the study, } \\
\text { the importance of study findings, and the take-home-message); Address to potential readers (i.e., } \\
\text { indicating why the journal's readers would be interested in this study) }\end{array}$ \\
\hline Third paragraph (declarations/obligatory statements) & $\begin{array}{l}\text { Author agreement statement; Conflict of interest statement; Funding source declaration; Ethical } \\
\text { standards or other statements required by the journal; Permission statement (if the manuscript } \\
\text { contains previously published materials); Statement regarding English native editing (if applicable); } \\
\text { Potential reviewers }\end{array}$ \\
\hline Cover letter footer (closing salutation) & $\begin{array}{l}\text { "Sincerely," or "Best regards," or ...; Sign of corresponding author (s) (with full name and academic } \\
\text { position); Contact information of corresponding author and co-authors (if required by the journal) } \\
\text { (i.e., affiliation, postal address, email address) }\end{array}$ \\
\hline
\end{tabular}

eration for publication elsewhere" (8). Some journals may request the corresponding author to confirm that he/she will take responsibility for informing co-authors of editorial decisions, reviews received, and any changes or revisions made; additionally, the editor (s) should be informed about any closely related manuscript (s) simultaneously submitted for consideration to the same or another journal (15). The authors also should declare if any part of the submitted work has been previously published elsewhere, even as an abstract (16); e.g., "there is some overlap in the content of the introduction section, which we have noted in the text".

Depending on the journal's policy, other statements, including "conflict of interest statement", "funding source declarations", and “permission note”, may also be required to be included in the cover letter $(8,11)$. As indicated by Elsevier, a conflict of interest statement, known as a disclosure statement, is a declaration from the author that "there is no financial/personal interest or belief that could affect their objectivity". The publisher emphasizes that the authors should declare and state the potential conflict's source and nature in cases where a conflict of interest exists. A funding source declaration is defined by the publisher as "a declaration of any funding or research grants (and their sources) received in the course of study, research or assembly of the manuscript". Elsevier also defines the permission note as a statement that declares that "permission has been received to use any material in the manuscript such as a figure, which is not original content" $(8,17)$. Other statements like "Statement of English native editing" may also be added.

Furthermore, informing the editor (s) regarding any information that will support the submission (e.g., original or confirmatory data, supplementary materials, rel- evance, topicality) can be helpful (8). Other operational information, typically provided within checkboxes of the journal's submission system, is not required to be included in the cover letter (5).

\subsection{Second Cover Letter}

The second cover letter, which accompanies the revised version of the manuscript, must be a model of clarity and must address every issue posed by the editor and reviewers (10). If the revised manuscript is sent for the second round of peer-review, the reviewer (s) will see the letter. The content of the header and footer sections of the revised letter is similar to that of the submitted cover letter. The letter should be directed to the editor as addressed in the first letter unless the authors are informed that a new editor will process the revised version (10). The first paragraph should start with an "expression of polite gratitude", e.g., "we would like to thank you for the opportunity to revise and resubmit our manuscript." The "manuscript ID" or "identification number," usually assigned by the journal in the first submission, should be addressed in the first paragraph (10).

The second paragraph usually "signals attention to the reviewers' comments" by providing an explicit reference to the comments made by the reviewers and the editor. Furthermore, it may contain a positive statement regarding the results, methodology, conclusions, etc., in which case the authors need to acknowledge reviews' insights $(10,18)$. For example, "We sincerely appreciate all the valuable comments and suggestions made, which helped us improve the revised version of our manuscript" or "we found the reviewers' comments helpful in guiding us to revise the manuscript." Such statements will help the authors in creating a polite, formal tone throughout the let- 
ter. The paragraph should be followed by providing the editor with a roadmap or a summary of the revisions, addressing "the response to comments attachment." A pointby-point response to the specific comments of the reviewers must be provided. If the authors disagree with a point raised by a reviewer, a rebuttal or counterstatement may be in order. A scientific and polite approach should spell out why the authors disagree, never losing sight of the reviewer's opinion (19).

The footer section (closing salutation) of the letter returns to polite formalities, using statements like "we hope that the revised version of our manuscript is now acceptable to the reviewers, and suitable for publication in the [name of journal], we look forward to hearing from you at your earliest convenience" (10).

\section{Organization}

Although it is not a rule, the cover letter's content can be organized within a cover letter header (opening salutation), three main paragraphs (the body of cover letter), and a cover letter footer (closing salutation), as described in Table 1.

The cover letter should be initiated by addressing the editor (s) and the target journal; however, the author's affiliation and contact information may also be included at the top of page (4). The name of the editor (s) can be easily found on the journal's information page. If it is known, the authors must address the editor who will receive the manuscript and handle the peer-review process (13). If there are several co-editors, the person the author feels has the most appropriate background, and specialty of the topic should be addressed. In cases where such information is lacking, authors can mention all editors by name or address the letter to "dear editors" (12); however, it has been recommended to avoid writing "dear editor" (16). Also, the submission date and the journal's name where the manuscript will be submitted are required (13).

In the first paragraph of the cover letter body, to introduce the submitted work, the title and the type of manuscript, authors' name, journal name, and manuscript length are presented (4). In addition, it is mentioned that whether the manuscript is submitted upon an invitation or belongs to a special issue. The importance of the study, including novelty, potential implications, and its take-home message, are addressed in the second paragraph of the cover letter body. In addition, it is explained why the work would be attractive for journal readers. The third paragraph of the cover letter body includes some statements including authorship agreement, conflicts of interest, funding source, and ethical considerations. If required, potential reviewers are also suggested here.

Within the closing salutation, the authors can appreciate the editor for taking the time to read the cover letter and considering the submitted work for potential publication.

\section{Some Practical Tips: The Length, and Dos and Don'ts}

The authors need to spend plenty of time crafting their cover letters. They are advised to avoid too many details and keep it within one page (less than 200 words), like an introduction or a brief overview $(4,11)$. The authors should check the guide for authors and cover letter suggestions provided by the journal, including all the requirements, e.g., specific disclosures, statements, and potential reviewers. Some publishers (e.g., Springer, https://www.springer.com/gp/authorseditors/journal-author/cover-letters/1398, Taylor \& Francis, https:/authorservices.taylorandfrancis.com/publishingyour-research/making-your submission/writing-a-journalarticle-cover-letter/) provide sample cover letters that the authors can use. Figure 1 provides a sample for a cover letter.

\subsection{Dos}

If the authors address previously published papers in the cover letter, then appropriate citation should be considered. The authors should carefully check the letter for any spelling and grammatical errors $(11,20)$. They should make sure that they correctly spell the name of the journal's editor (s) (4). Being careless regarding the editor's name or the change of a journal's name in a cover letter of a resubmitted manuscript, can be embarrassing and make a bad impression (4). It is suggested that the cover letter be written on the authors' institutional letterhead to display professionalism and reliability $(20,21)$.

\subsection{Don'ts}

When authors suggest a number of potential reviewers, they should avoid suggesting their friends and colleagues, as this would be viewed as a conflict of interest. Collaborators whom the authors have published with in the past five years should not be suggested either; an editor may easily be informed of such associations by a quick search of PubMed or other databases (22). The authors should avoid using complex sentence structures, jargon, and acronyms and keep the text straightforward and easy to read $(11,20)$. The authors should also avoid including unrelated personal information or glorifying their past research papers or any of their academic accolades (20). 


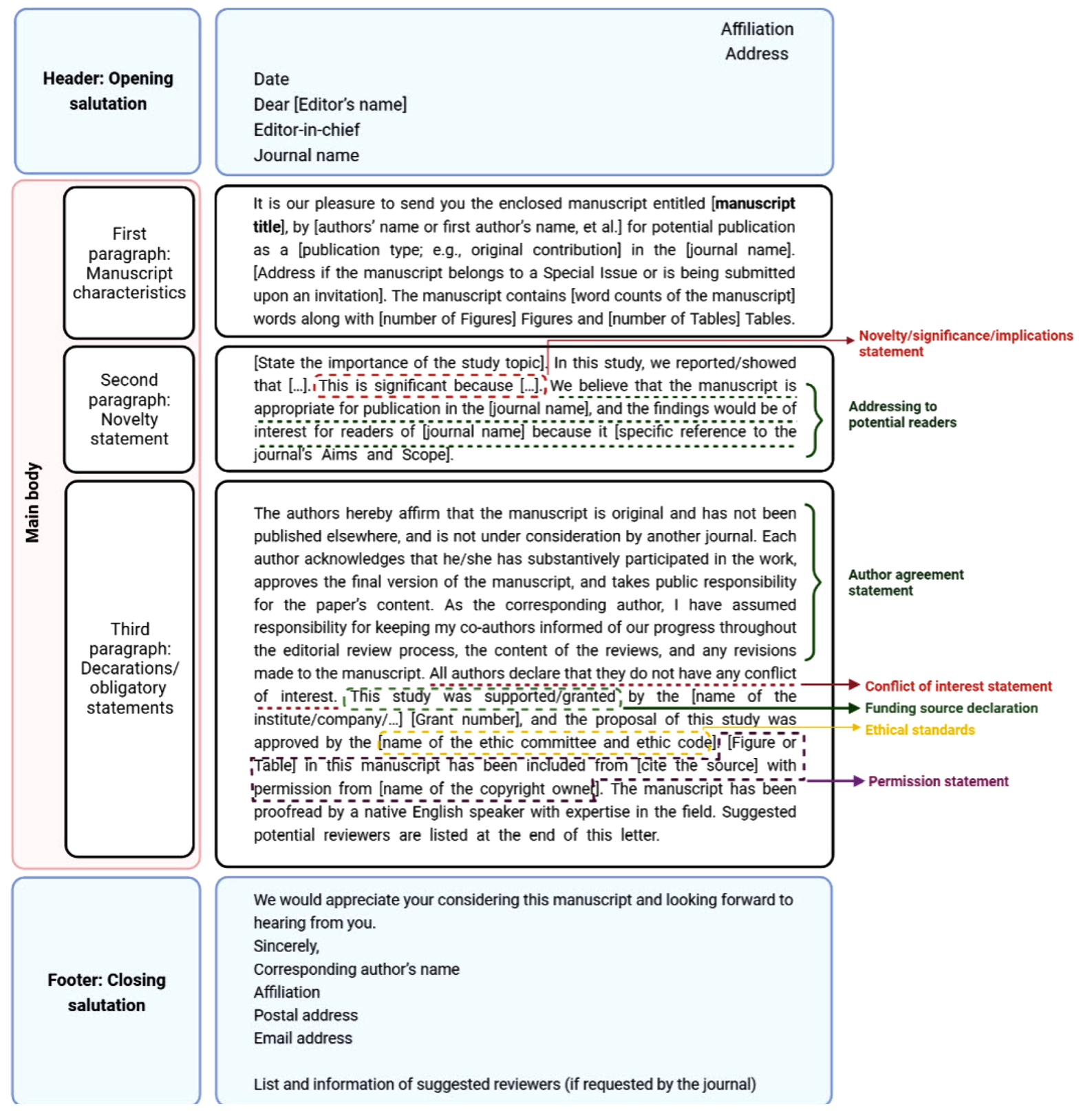

Figure 1. A sample for a cover letter.

They must not be rude towards the editors or complement the editor's accomplishments (4). The novelty statement should not exaggerate or overstate the findings of the work; furthermore, any conclusion stated should be completely supported by the data provided in the manuscript (23). Finally, authors are recommended not to write a generic cover letter that could be used for any manuscript and could be sent to any journal (21).

\section{Conclusion}

In summary, a cover letter should highlight the novelty, importance, take-home message, and goodness-of-fit of the manuscript to the journal. These are critical information that can persuade an editor that the submitted work merits publication consideration in the journal. The cover letter should not be general but should be customwritten for the target journal. Although the submitted 
manuscript may usually pass through the peer-review process and get published regardless of the cover letter, a wellwritten, informative, and concise cover letter increases the chance of gaining acceptance.

\section{Footnotes}

Authors' Contribution: Study concept and design, Zahra Bahadoran and Asghar Ghasemi; Drafting of the manuscript, Zahra Bahadoran, Parvin Mirmiran, and Asghar Ghasemi; Critical revision of the manuscript for important intellectual content, Khosrow Kashfi and Parvin Mirmiran.

Conflict of Interests: The authors have no conflict of interest.

Funding/Support: This study was supported by the Shahid Beheshti University of Medical Sciences (grant number 28127).

\section{References}

1. Beatty RH. 175 high-impact cover letters. New Jersey, USA: J. Wiley; 1996.

2. Tomaska L, Nosek J. Ten simple rules for writing a cover letter to accompany a job application for an academic position. PLoS Comput Biol. 2018;14(5). e1006132. doi: 10.1371/journal.pcbi.1006132. [PubMed: 29851981]. [PubMed Central: PMC5978783].

3. Moustafa K. Does the cover letter really matter? Sci Eng Ethics. 2015;21(4):839-41. doi: 10.1007/s11948-014-9554-8. [PubMed: 24839946].

4. John M. I really think you should publish this paper: the cover letter to the editor. HSR Proc Intensive Care Cardiovasc Anesth. 2011;3(2):137-9. [PubMed: 23441272]. [PubMed Central: PMC3484622].

5. Yates JR. The cover letter. J Proteome Res. 2017;16(2):367. doi: 10.1021/acs.jproteome.6b01068. [PubMed: 28055218].

6. Hafner JH. The art of the cover letter. ACS Nano. 2010;4(5):2487. doi: $10.1021 /$ nn100907e.

7. Bahadoran Z, Mirmiran P, Kashfi K, Ghasemi A. Scientific publishing in biomedicine: How to choose a journal? Int J Endocrinol Metab. 2021;19(1). e108417. doi: 10.5812/ijem.108417. [PubMed: 33815519]. [PubMed Central: PMC8010430].

8. Elsevier. What should be included in a cover letter? Amsterdam, Netherlands: Elsevier; 2021, [updated 7th Jun 2021; cited 15th Mar 2021]. Available from: https://service.elsevier.com/app/answers/detail/a $\mathrm{id} / 155 /$ supporthub/publishing/ /what-should-be-included-in-acover-letter\%3F/.

9. Körner AM. First letter to the editor of your target journal. In: Körner AM, editor. Guide to publishing a scientific paper Routledge. London, UK: Routledge; 2008. p. 83-7. doi: 10.4324/9780203938751.
10. Körner AM. Second letter to the editor of your target journal. In Körner AM, editor. Guide to publishing a scientific paper Routledge. London, UK: Routledge; 2008. p. 95-9. doi: 10.4324/9780203938751.

11. Taylor \&amp; Francis Group. How to write a cover letter for journal article submission. London, UK: Informa Group Company; 2021, [cited 15th Mar 2021]. Available from: https://authorservices.taylorandfrancis. com/publishing-your-research/making-your-submission/writinga-journal-article-cover-letter/\#.

12. Nicholas D. How to choose a journal and write a cover letter. Saudi J Anaesth. 2019;13(Suppl 1):S35-41. doi:10.4103/sja.SJA_691_18. [PubMed: 30930718]. [PubMed Central: PMC6398290].

13. Springer. Cover letters. Switzerland: Springer; 2021, [cited 14th Mar 2021]. Available from: https://www.springer.com/gp/authorseditors/authorandreviewertutorials/submitting-to-a-journal-andpeer-review/cover-letters/10285574.

14. Sacristán C. Submitting your manuscript: Write the right cover letter. Amsterdam, Netherlands: Elsevier Inc; 2017, [updated 24th Jan 2017 cited 5th Apr 2021]. Available from: http://crosstalk.cell.com/blog/ submitting-your-manuscript-write-the-right-cover-letter.

15. APA Style. Cover letters. Washington, USA: American Psychological Association; 2019, [cited 5th Apr 2021]. Available from: https://apastyle.apa.org/style-grammar-guidelines/researchpublication/cover-letters.

16. Reis R. Write a persuasive cover letter (for your research paper submission). California, USA: Stanford University; 2021, [cited Apr 2021]. Available from: https://tomprof.stanford.edu/posting/1566.

17. Elsevier. What are conflict of interest statements, funding source declarations, author agreements/declarations and permission notes? Amsterdam, Netherlands: Elsevier; 2021, [updated 7th Jun 2021; cited 12th Mar 2021]. Available from: https://service.elsevier.com/app/answers/ detail/a_id/286/supporthub/publishing/related/1/session/.

18. Student Academic Success Center. Writing the revise and resubmit letter Pennsylvania, USA: Carnegie Mellon University; 2021, [cited 4th Apr 2021]. Available from: https://www.cmu.edu/student-success/otherresources/resource-descriptions/revise-and-resubmit.html.

19. Springer. Revising and responding. Switzerland: Springer Nature; 2021, [cited 4th Apr 2021]. Available from: https://www.springer.com/ gp/authors-editors/authorandreviewertutorials/submitting-to-ajournal-and-peer-review/revising-and-responding/10285584.

20. Chan A. How to write a cover letter when submitting your research paper London, UK: Dove Press Ltd; 2018, [updated 3rd Jul 2018; cited 15th Mar 2021]. Available from: https://www.dovepress.com/how-to-write-acover-letter-blog-post\#.

21. Corriveau M, Lake W, Hanna A. Nerve stimulation for pain. Neurosurg Clin N Am. 2019;30(2):257-64. doi: 10.1016/j.nec.2018.12.008. [PubMed 30898276].

22. Sahni P, Aggarwal R. Reporting and publishing research in the biomedical sciences. Switzerland: Springer Nature; 2018.

23. BioScience Writers. Writing cover letters for scientific manuscripts Texas, USA: BioScience Writers; 2021, [cited 4th Apr 2021]. Available from: https://www.biosciencewriters.com/Writing-Cover-Lettersfor-Scientific-Manuscripts.aspx. 